\title{
A GENERALIZATION OF TWO INEQUALITIES INVOLVING MEANS ${ }^{1}$
}

\author{
SCOTT LAWRENCE AND DANIEL SEGALMAN
}

\begin{abstract}
Fan has proven an inequality relating the arithmetic and geometric means of $\left(x_{1}, \cdots, x_{n}\right)$ and $\left(1-x_{1}, \cdots, 1-x_{n}\right)$, where $0<x_{i} \leqq \frac{1}{2}, i=1, \cdots, n$. Levinson has generalized Fan's inequality; his result involves functions with positive third derivatives on $(0,1)$. In this paper, the above condition that requires $0<x_{i} \leqq \frac{1}{2}$ has been replaced by a condition which only weights the $x_{i}$ to the left side of $(0,1)$ in pairs, and Levinson's differentiability requirement has been replaced by the analogous condition on third differences.
\end{abstract}

1. Introduction. Levinson [1] has generalized the following inequality of Ky Fan:

Let $0<x_{i} \leqq \frac{1}{2}, i=1, \cdots, n$. Then

$$
\prod_{i=1}^{n} x_{i} /\left(\sum_{i=1}^{n} x_{i}\right)^{n} \leqq \prod_{i=1}^{n}\left(1-x_{i}\right) /\left(\sum_{i=1}^{n}\left(1-x_{i}\right)\right)^{n}
$$

with equality if and only if all the $x_{i}$ are equal.

Levinson's result is the following:

Let $f$ have a third derivative on $(0,2 a)$ with $f^{\prime \prime \prime}(x) \geqq 0$ for all $x$ in $(0,2 a)$. If $0<x_{i} \leqq a$ and $0<p_{i}, i=1, \cdots, n$, then

$$
\begin{aligned}
\sum_{i=1}^{n} p_{i} f\left(x_{i}\right) / \sum_{i=1}^{n} p_{i}-f\left(\sum_{i=1}^{n} p_{i} x_{i} / \sum_{i=1}^{n} p_{i}\right) \\
\leqq \sum_{i=1}^{n} p_{i} f\left(2 a-x_{i}\right) / \sum_{i=1}^{n} p_{i}-f\left(\sum_{i=1}^{n} p_{i}\left(2 a-x_{i}\right) / \sum_{i=1}^{n} p_{i}\right) .
\end{aligned}
$$

Furthermore, if $f^{\prime \prime \prime}(x)>0$ on $(0,2 a)$, then the above inequality reduces to equality if and only if all the $x_{i}$ are equal.

Ky Fan's inequality is Levinson's inequality with $p_{i}=1, a=\frac{1}{2}$, and $f(x)=\log x$. Popoviciu [2] has slightly weakened the differentiability assumption in Levinson's theorem.

Presented to the Society, November 27, 1971 under the title Generalization of an inequality of Levinson and Fan; received by the editors October 15, 1971.

AMS 1970 subject classifications. Primary 26A86.

${ }^{1}$ Supported by the National Science Foundation. 
Our purpose is to prove Levinson's inequality and certain special cases of it under more natural, less restrictive conditions than the above.

2. Main result. The following theorem extends Levinson's inequality in the case of equal weights.

THEOREM 1. Let $f$ be a continuous function defined on $(0,2 a)$ for which $\Delta_{h}^{3} f(x)>0$ for all $x$ in $(0,2 a)$ and $h>0$ for which $\Delta_{h}^{3} f(x)$ is defined (i.e., for all $x$ in $(0,2 a)$ and $h>0$ for which $x+3 h<2 a)$. Let $x_{1}, \cdots, x_{n}$ be numbers in $(0,2 a)$ such that $x_{1} \leqq x_{2} \leqq \cdots \leqq x_{n}$ and $x_{i}+x_{n+1-i} \leqq 2 a, i=1, \cdots, n$. Then

(2) $\frac{1}{n} \sum_{i=1}^{n} f\left(x_{i}\right)-f\left(\frac{1}{n} \sum_{i=1}^{n} x_{i}\right) \leqq \frac{1}{n} \sum_{i=1}^{n} f\left(2 a-x_{i}\right)-f\left(\frac{1}{n} \sum_{i=1}^{n}\left(2 a-x_{i}\right)\right)$

with equality if and only if either all the $x_{i}$ are equal or $x_{i}+x_{n+1-i}=2 a$, $i=1, \cdots, n$.

Several remarks are in order concerning the statement of the theorem. First of all, the order of the $x_{i}$ is obviously irrelevant. We merely need to be able to pair them in such a manner that the sum of the members of each pair is at most $2 a$. The ordering condition in the theorem is motivated entirely by notational convenience. Secondly, if $f$ is continuous and $\Delta_{h}^{3} f(x)>0$ for all $x$ and $h>0$, then $\Delta_{j} \Delta_{k} \Delta_{l} f(x)>0$ for all $x$ and $j, k, l>0$ (a direct proof is not immediate, but is fairly easy). In the proof of the theorem we shall only need that $\Delta_{k}^{2} \Delta_{h} f(x)>0$. Of course, if $f$ is three times differentiable and $f^{\prime \prime \prime}(x)>0$ for all $x$ in $(0,2 a)$, then third differences are also positive. Conversely, if third differences are positive, then $f^{\prime \prime \prime}(x)$ is nonnegative (but not necessarily strictly positive). The third and probably most important feature of the theorem different from Levinson's theorem is that the $x_{i}$ are somewhat less restricted. Finally, if we retain Levinson's restriction on the $x_{i}$, i.e. $0<x_{i} \leqq a$, then the theorem for arbitrary weights can be proven as an easy corollary to the theorem for equal weights. Theorem 1 can be proven under the assumption that $\Delta_{h}^{3} f(x) \geqq 0$ as a limiting case of the theorem as stated above, but the cases of equality are then obscured.

ProOF OF THE THEOREM. If all the $x_{i}$ are equal, both sides of the inequality reduce to zero. If $x_{i}+x_{n+1-i}=2 a$ for all $i$, then the right side is the same as the left except for a reversal in the order of the summations.

Suppose not all the $x_{i}$ are equal and that they are not summable in pairs of $2 a$. Furthermore, in the pairing hypothesis assume first that some pair (let it be $\left(x_{1}, x_{n}\right)$ for convenience) has sum less than $2 a$ and has unequal members. Then let $h=2 a-x_{n}-x_{1}, k=\left(x_{n}-x_{1}\right) / 2$. Since $x_{1}+x_{n}<2 a$ 
and $x_{1}<x_{n}$, we have $h>0, k>0$. Thus,

Hence,

$$
\begin{aligned}
0< & \Delta_{k}^{2} \Delta_{h} f\left(x_{1}\right) \\
= & f\left(x_{1}+h+2 k\right)-2 f\left(x_{1}+h+k\right) \\
& +f\left(x_{1}+h\right)-f\left(x_{1}+2 k\right)+2 f\left(x_{1}+k\right)-f\left(x_{1}\right) \\
= & f\left(2 a-x_{1}\right)-2 f\left(2 a-\left(x_{1}+x_{n}\right) / 2\right)+f\left(2 a-x_{n}\right) \\
& \quad-f\left(x_{n}\right)+2 f\left(\left(x_{1}+x_{n}\right) / 2\right)-f\left(x_{1}\right) .
\end{aligned}
$$

$$
\begin{aligned}
2 f\left(\left(x_{1}+x_{n}\right) / 2\right)- & 2 f\left(2 a-\left(x_{1}+x_{n}\right) / 2\right) \\
> & f\left(x_{1}\right)+f\left(x_{n}\right)-f\left(2 a-x_{1}\right)-f\left(2 a-x_{n}\right) .
\end{aligned}
$$

Thus we have proven that replacing each of $x_{1}$ and $x_{n}$ by $\left(x_{1}+x_{n}\right) / 2$ increases the function

$$
l\left(x_{1}, \cdots, x_{n}\right)=\frac{1}{n}\left(\sum_{i=1}^{n} f\left(x_{i}\right)-\sum_{i=1}^{n} f\left(2 a-x_{i}\right)\right) .
$$

Given any $n$-tuple of real numbers $\left(c_{1}, \cdots, c_{n}\right)$ subject to the conditions $0<c_{i}<2 a, c_{i}+c_{n+1-i} \leqq 2 a, i=1, \cdots, n$, and $c_{1} \leqq c_{2} \leqq \cdots \leqq c_{n}$, we consider the compact set $D \subseteq R^{n}$ defined as follows: $\left(x_{1}, \cdots, x_{n}\right)$ is in $D$ if and only if

$$
\begin{gathered}
c_{1} \leqq x_{1} \leqq x_{2} \leqq \cdots \leqq x_{n} \leqq c_{n} \\
x_{1}+x_{2}+\cdots+x_{n}=c_{1}+c_{2}+\cdots+c_{n} \\
x_{i}+x_{n+1-i} \leqq 2 a, \quad i=1, \cdots, n
\end{gathered}
$$

Since $l\left(x_{1}, \cdots, x_{n}\right)$ is continuous on $D$, it takes on a maximum value there. Let $l\left(y_{1}, \cdots, y_{n}\right)$ be this maximum for some $\left(y_{1}, \cdots, y_{n}\right)$ in $D$. We have that either $y_{1}=y_{2}=\cdots=y_{n}$ or $y_{i}+y_{n+1-i}=2 a, i=1, \cdots, n$, as follows. If there is some $j$ for which $y_{j} \neq y_{n+1-j}$ and $y_{j}+y_{n+1-j}<2 a$, then replacing $y_{j}$ and $y_{n+1 \cdots j}$ by $\left(y_{j}+y_{n+1-j}\right) / 2$ and renumbering, we would obtain an $n$-tuple (in $D)\left(z_{1}, \cdots, z_{n}\right)$ for which $l\left(z_{1}, \cdots, z_{n}\right)>l\left(y_{1}, \cdots, y_{n}\right)$, as above. Hence, there are no maximizing $n$-tuples with these properties, so for each $i$ either $y_{i}=y_{n+1-i}$ or $y_{i}+y_{n+1-i}=2 a$.

Suppose this is the case. We know that

$$
r\left(x_{1}, \cdots, x_{n}\right)=f\left(\frac{1}{n} \sum_{i=1}^{n} x_{i}\right)-f\left(\frac{1}{n} \sum_{i=1}^{n}\left(2 a-x_{i}\right)\right)
$$

remains constant on $D$. We shall show that unless all the pairs of the $x_{i}$ sum to $2 a$ or all the $x_{i}$ are equal, then $l\left(x_{1}, \cdots, x_{n}\right)<r\left(x_{1}, \cdots, x_{n}\right)$. Since equality holds when $y_{1}=y_{2}=\cdots=y_{n}=\left(c_{1}+\cdots+c_{n}\right) / n\left(\left(y_{1}, \cdots, y_{n}\right)\right.$ 
is in $D$ in this case), we will have eliminated all but the stated possible maximizing $n$-tuples.

Suppose that for some $j$ we have that $y_{j}=y_{j+1}=\cdots=y_{n+1-j}$ (if $y_{j}=$ $y_{n+1-j}$, this follows from the ordering), that $y_{j}<a$, and that $y_{i}+y_{n+1-i}=2 a$ for all $i<j$. Then

$$
\begin{aligned}
& \frac{1}{n} \sum_{i=1}^{n} f\left(y_{i}\right)-\frac{1}{n} \sum_{i=1}^{n} f\left(2 a-y_{i}\right) \\
& \quad=\frac{1}{n} \sum_{i=j}^{n+1-j}\left(f\left(y_{i}\right)-f\left(2 a-y_{i}\right)\right)=\frac{n+2-2 j}{n}\left[f\left(y_{j}\right)-f\left(2 a-y_{j}\right)\right],
\end{aligned}
$$

and

$$
\begin{aligned}
f\left(\frac{1}{n} \sum_{i=1}^{n} y_{i}\right)-f\left(\frac{1}{n} \sum_{i=1}^{n}\left(2 a-y_{i}\right)\right) & =f\left(\frac{2 j-2}{n} a+\frac{n+2-2 j}{n} y_{j}\right) \\
& -f\left(\frac{n+2-2 j}{n}\left(2 a-y_{j}\right)+\frac{2 j-2}{n} a\right) .
\end{aligned}
$$

Let $\omega=(n+2-2 j) / n, \delta=a-y_{j}$. Consider

$$
\begin{array}{rl}
\frac{1}{n} \sum_{i=1}^{n} & f\left(2 a-y_{i}\right)-\frac{1}{n} \sum_{i=1}^{n} f\left(y_{i}\right)+f\left(\frac{1}{n_{i=1}} \sum_{i=1}^{n} y_{i}\right)-f\left(\frac{1}{n} \sum_{i=1}^{n}\left(2 a-y_{i}\right)\right) \\
= & \frac{n+2-2 j}{n}\left[f\left(2 a-y_{j}\right)-f\left(y_{j}\right)\right]+f\left(\frac{2 j-2}{n} a+\frac{n+2-2 j}{n} y_{j}\right) \\
& -f\left(\frac{n+2-2 j}{n}\left(2 a-y_{j}\right)+\frac{2 j-2}{n} a\right) \\
= & \omega[f(a+\delta)-f(a-\delta)]+[f(a-\omega \delta)-f(a+\omega \delta)]=G(\omega) .
\end{array}
$$

We would like to show that $G(\omega)>0$ unless either all of the $y_{i}$ are equal $(\omega=1)$, or $y_{i}+y_{n+1-i}=2 a, i=1, \cdots, n(\omega=0)$. We know that $G(0)=0=$ $G(1)$. Let $c$ and $d$ be any strictly positive numbers. Then a simple observation reveals that $\Delta_{c} \Delta_{d} G(\omega)=-\Delta_{c} \Delta_{d} \Delta_{2 \omega \delta} f(a-\omega \delta)<0$ if $\omega$ is in $(0,1)$ and either difference is defined. Hence, $G(\omega)$ is a strictly concave function on $(0,1)$ with $G(0)=0=G(1)$, so $G(\omega)>0$ for $\omega$ in $(0,1)$. This proves that the only cases of equality are those stated above, so the proof of the theorem is complete.

3. Integral generalization. By similar methods we obtain the following generalization.

THEOREM 2. Let $f$ be a continuous function on $(0,2 a)$ for which $\Delta_{h}^{3} f(x)>0$ for all $x$ in $(0,2 a)$ and $h>0$, and let $g$ be an integrable positive 
function on $(0,1)$ such that $g(t)+g(1-t) \leqq 2 a$ for all $t$ in $(0,1)$. Then

$$
\begin{aligned}
\int_{0}^{1} f(g(t)) d t- & f\left(\int_{0}^{1} g(t) d t\right) \\
& \leqq \int_{0}^{1} f(2 a-g(t)) d t-f\left(\int_{0}^{1}(2 a-g(t)) d t\right)
\end{aligned}
$$

with equality if and only if either $g(t)$ is constant almost everywhere on $(0,1)$ or $g(t)+g(1-t)=2 a$ almost everywhere on $(0,1)$.

This theorem can be specialized to a generalized version of (2) as follows. Let $\left\{p_{i}\right\}$ and $\left\{x_{i}\right\}$ be sequences of $n$ positive real numbers with $x_{i}<2 a, i=1, \cdots, n$. Define $p_{0}=0, g(t)=x_{j}$ for $\sum_{i=0}^{j-1} p_{i} / \sum_{i=0}^{n} p_{i}<t \leqq$ $\sum_{i=0}^{j} p_{i} / \sum_{i=0}^{n} p_{i}$, and suppose that $\left\{p_{i}\right\}$ and $\left\{x_{i}\right\}$ are such that $g(t)+g(1-t) \leqq$ $2 a$ for all $t$ in $(0,1)$. Then, inequality (3) yields

$$
\begin{aligned}
\sum_{i=1}^{n} p_{i} f\left(x_{i}\right) / \sum_{i=1}^{n} & p_{i}-f\left(\sum_{i=1}^{n} p_{i} x_{i} / \sum_{i=1}^{n} p_{i}\right) \\
& \leqq \sum_{i=1}^{n} p_{i} f\left(2 a-x_{i}\right) / \sum_{i=1}^{n} p_{i}-f\left(\sum_{i=1}^{n} p_{i}\left(2 a-x_{i}\right) / \sum_{i=1}^{n} p_{i}\right),
\end{aligned}
$$

with equality if and only if either all the $x_{i}$ are equal or $g(t)+g(1-t)=2 a$ for all but a finite set of $t$.

Inequality (4) essentially generalizes (1) to the case where the means are weighted to the left. If we retain Levinson's restriction on the $\left\{x_{i}\right\}$, (4) yields (1).

ACKNOWLEDGEMENTS. The authors wish to express their thanks to Professor Richard Askey for bringing up the problem and for his very useful suggestions and comments during the development of the paper, and to Behzad Razban for his comments.

\section{REFERENCES}

1. N. Levinson, Generalization of an inequality of $K y$ Fan, J. Math. Anal. Appl. 8 (1964), 133-134. MR 28 \#171.

2. T. Popoviciu, Sur une inégalité de N. Levinson, Mathematica (Cluj) 6 (1964), 301306.

Department of Mathematics, University of Wisconsin, Madison, Wisconsin 53706 